\title{
Características sociodemográficas y condiciones de vida y estudio de los estudiantes de Educación de origen indígena. La aproximación del proyecto TO-INN ${ }^{1}$
}

\author{
Socio-Demographic Characteristics and Living Conditions and Study \\ of Indigenous Education Students. The Approach of the TO-INN Project
}

Jordi Méndez Ulrich, Alejandra Montané López, Juan Llanes Ordoñez, Gabriel Hervas Nicolás, Isaac Calduch Pérez, Ricardo Morales-Ulloa y Judith Muñoz Saavedra ${ }^{2}$

\section{Resumen}

Uno de los objetivos principales del proyecto From Tradition to Innovation in Teacher Training Institutions (TO-INN), ha consistido en conocer a los estudiantes de educación a través del análisis de la dimensión social de las instituciones de Educación Superior. El presente trabajo pretende establecer un debate sobre la presencia de estudiantes indígenas en las universidades, y más concretamente, en los estudios de educación. El proyecto TO-INN parte de una visión sistémica del compromiso social de la Educación Superior con los colectivos tradicionalmente excluidos y especialmente vulnerables, como los pueblos indígenas, que a pesar de haber aumentado el porcentaje de accesoa la universidad convive con desigualdades relacionadas con la inclusión, la permanencia/supervivencia, las condiciones de vida, la participación y los resultados académicos. En este trabajo presentamos: a) una revisión teórica y contextual sobre los pueblos indígenas y su relación con la Educación Superior; b) el perfil sociodemográfico y académico de los 701 estudiantes de carreras del ámbito educativo pertenecientes a comunidades indígenas o pueblos originarios que participaron en el cuestionario TO-INN sobre la dimensión social (sobre una muestra total de 12238 participantes); c) la distribución por países de los estudiantes indígenas participantes; d) los datos universitarios de dichos estudiantes; e) su contexto familiar; f) sus condiciones de vida, y g) la participación académica y social de este colectivo. Para finalizar, se presentan las limitaciones del estudio y posibles líneas de investigación futura.

\section{Palabras clave}

Estudiantes indígenas, educación superior, América Latina, dimensión social.

\section{Abstract}

One of the principle objectives of the project, From Tradition to Innovation Teacher Training Institutions (TO-INN), was the analysis of the social dimension of students of education in Higher Education institutions. This work aims to stimulate a debate on the presence of indigenous students in universities, specifically in education studies. The TO-INN project is the result of a systemic vision of the social commitment of Higher Education with groups which have traditionally been at risk of exclusion and vulnerability. In particular, the study focuses on the analysis of indigenous peoples. Despite the percentage of those who Access University having increased, inequalities related to inclusion, persistence and survival, living conditions, participation and academic results persist. In this paper we present: a) a theoretical and contextual review of the indigenous peoples and their relationship with Higher Education; b) the socio-demographic and academic profile of the 701 students of courses in the educational field belonging to indigenous communities or native peoples who undertook the TO-INN questionnaire on the social dimension from a total sample of 12,238 participants; c) the distribution by country of the participating indigenous students; d) the information held by the university on those students ; e) their family context ; f) their living conditions, and g) the academic and social participation of this group. Finally, the limitations of the study are explained, and suggestions for possible future lines of research.

\section{Keywords}

Indigenous students, higher education, Latin America, social dimension.

\section{Cómo citar/Citation}

Méndez Ulrich, Jordi; Montané López, Alejandra; Llanes Ordoñez, Juan; Hervas Nicolás, Gabriel; Calduch Pérez, Isaac; Morales-Ulloa, Ricardo; Muñoz Saavedra, Judith (2019). Características sociodemográficas y condiciones de vida y estudio de los estudiantes de Educación de origen indígena. La aproximación del proyecto TO-INN. Revista de Sociología de la Educación-RASE, 12 (2), 139-156. http://dx.doi.org/10.7203/RASE.12.2.14810.

\footnotetext{
1 Este artículo forma parte del proyecto TO-INN From Tradition to Innovation in Teacher Training Institutions financiado por la Unión Europea a través del programa Erasmus, Acción K2 Capacity Building, (573685-EPP-2016-1-ES-EPPKA2-CBHE-JP) https://www.toinn.org.

2 Jordi Méndez Ulrich, jordi.mendez@ub.edu; Alejandra Montané López, smontane@ub.edu; Juan Llanes Ordoñez, juanllanes@ub.edu; Gabriel Hervas Nicolás, ghervas@ ub.edu; Isaac Calduch Pérez, icalduch@ub.edu; Judith Muñoz Saavedra, judithmunoz@ub.edu. Universitat de Barcelona. Ricardo Morales-Ulloa, Universidad Pedagógica Nacional Francisco Morazan, rmorales@upnfm.edu.hn;
} 


\section{Introducción}

Este estudio se enmarca en un proyecto internacional en curso que pretende tender puentes desde la tradición hacia la innovación en instituciones de Educación Superior en América Latina y Europa. Bajo el acrónimo TO-INN (From Tradition to innovation in Teacher Training Institutions), este proyecto parte de una visión sistémica del compromiso social en Educación Superior que se relaciona con las tendencias actuales que consideran que los sistemas e instituciones de Educación Superior deben fortalecer las perspectivas sociales, entendiendo la complejidad y multidimensionalidad de los problemas y favoreciendo la transversalidad de los enfoques que la preceden (Henríquez, 2018).

En este sentido, entendemos que el proceso de democratización y masificación de la Educación Superior convive, paradójicamente, con desigualdades relacionadas con la inclusión, el acceso, la permanencia en la institución educativa, las condiciones de vida, la participación académica y social y los resultados académicos de los estudiantes. Por ello, entendemos que debe analizarse la compleja relación entre los/ las estudiantes, sus características y la dimensión social de la universidad que, más allá de la visión tradicional, focalizada en la composición social de la matrícula universitaria (Ariño, 2014), debe responder a su responsabilidad delimitada como la tercera misión de la universidad y que da lugar a la Responsabilidad Social Universitaria.

En este contexto, se considera que para poder hablar de innovación en las instituciones de Educación Superior y para mejorar las políticas que afectan a los estudiantes, siempre con la finalidad de avanzar hacia una Educación Superior más equitativa e inclusiva, no podemos obviarla importancia de seguir estudiando sus condiciones de vida, de participación, de posibilidades de supervivencia académica y de estudio, en especial, al considerar que en el contexto global actual, neoliberal y conservador, se atenta contra el principio de equidad participativa.

En el presente trabajo, y teniendo en cuenta que uno de los objetivos del estudio de la dimensión social es la participación en la Educación Superior de grupos especialmente vulnerables o históricamente excluidos, hemos centrado nuestra atención en el colectivo de estudiantes indígenas de América Latina que estudian en este tipo de instituciones convencionales que significa, utilizando la terminología de Mato (2008), instituciones no diseñadas específicamente para ellos.

Nuestra preocupación se encuentra alineada con el punto 4.5 de los Objetivos de Desarrollo Sostenible que destaca la necesidad de eliminar las disparidades de género y asegurar el acceso igualitario de las personas vulnerables, entre las que se encuentras aquellas procedentes de pueblos o comunidades indígenas (ONU, 2018).

\section{1. Situación de los pueblos indígenas en América Latina}

Para llegar a comprender la situación de los colectivos indígenas en la Educación Superior se debe recurrir a su historia y sus propios procesos identitarios, los cuales determinan sus relaciones con la educación y con los modos en que se crea y distribuye el conocimiento.

Este apartado, que no pretende ser exhaustivo, tiene como objetivo situar el colectivo indígena del territorio participante en la encuesta de un modo general y, particularmente, apuntar a su condición de colectivo vulnerable en el sistema educativo. Un colectivo que sufre una exclusión multidimensional entre los que se encuentra el propio sistema educativo que ha potenciado dicha situación de vulnerabilidad, actuando como un núcleo de inequidad (Didou, 2006; Robles 2009). 
Por una parte, «pueblos indígenas» podría constituirse como una categoría de análisis atravesada por procesos históricos, sociales y culturales altamente complejos. En el informe «zLos de afuera? La exclusión social en América Latina» se define como indígenas/pueblos indígenas a los habitantes precolombinos, sus descendientes y los diversos grupos étnicos que se identifican con esos pueblos históricos (IDEA, 2007).

En el ámbito internacional existe cierto consenso en la definición de «pueblos indígenas» a partir de los postulados de Martínez-Cobo (1986, citado por Del Popolo, 2008):

«Son comunidades, pueblos y naciones indigenas los que, teniendo una continuidad histórica con las sociedades anteriores a la invasión y colonización que se desarrollaron en sus territorios, se consideran a si mismos distintos de otros sectores de las sociedades que abora prevalecen en estos territorios o en partes de ellos. Constituyen ahora sectores no dominantes de la sociedad y tienen la determinación de preservar, desarrollar y transmitir a las generaciones futuras sus territorios ancestrales y su identidad étnica, como base de su existencia continuada como pueblos, de conformidad con sus propios patrones culturales, sus instituciones sociales y sus sistemas jurídicos». (E/CN.4 / Sub. 2 / 1986/7/Add. 4, párr. 379).

En esta definición se condensan así cuatro dimensiones centrales de la definición de pueblo indígena (Del Popolo, 2008a) reconocimiento de la identidad: se refiere al sentido de pertenencia al pueblo; b) origen común: descendencia de ancestros comunes e implica la memoria social y colectiva de los pueblos indígenas, entre otros factores; c) territorialidad: ligada a la herencia y ocupación de tierras ancestrales, así como a los vínculos materiales y simbólicos que se establecen con esta, y d) lingüístico-cultural: vinculada a la cultura de origen, una organización social y política determinada, el idioma, la cosmovisión, saberes, conocimientos y modos de vida.

En general se considera que los sujetos se definen como indígenas dado que se auto-identifican como descendientes de los pueblos que habitaban los territorios antes de la llegada de los conquistadores; y conforman pueblos que conservan sus instituciones sociales, económicas, culturales y políticas, total o parcialmente (Robles, 2009).

Según el informe de la Comisión Económica para América Latina (CEPAL, 2014) existe un problema histórico con los datos y estadísticas sobre pueblos indígenas. Con la excepción de tres países de América Latina (Cuba, Haití y República Dominicana). Otros han incluido la autoidentificación en los censos, o prevén hacerlo (Colombia, El Salvador, Nicaragua y Perú, por ejemplo, lo hicieron en sus censos de población de 2015 a 2017). Pero poco a poco ha aumentado la conciencia hacia el colectivo, se ha constatado en el aumento del número de países que incluyen preguntas relativas a los idiomas indígenas; en algunos casos, se trata de aquellos aprendidos en la niñez, en otros, de los idiomas que habla cada persona, o el que se utiliza más frecuentemente en el hogar.

Según dicho informe, en América Latina había cerca de 45 millones de indígenas en 2010, sumando aproximadamente el $8 \%$ de la población total y con una alta heterogeneidad según países: en un extremo están México, Guatemala, Bolivia y Perú, que concentran en 34\%del total regional y en el otro, Costa Rica y el Paraguay, con poco más de 100000 personas indígenas (CEPAL, 2014). No obstante, la situación por países difiere. En Bolivia y Guatemala la población indígena es mayoritaria; en México, Perú y Ecuador tienen una amplia diversidad de pueblos, pero no mayoría poblacional. 
Con respecto a la diversidad lingüística, López y Küper (1999) afirman que todavía sobreviven unas 500 lenguas indígenas, la mayoría en Brasil, México y Guatemala, aunque un buen número de ellas están consideradas en peligro. Esta tendencia a la desaparición se acelera ante el rápido avance de la globalización, advirtiendo acerca de cómo la vulnerabilidad socioeconómica y la debilidad del reconocimiento de los derechos de los pueblos indígenas son factores endógenos que debilitan el patrimonio lingüístico de los países latinoamericanos (FUNPREIB-Andes, 2009).

La pobreza no es una condición intrínseca de los pueblos indígenas, sino la consecuencia de una larga historia de agresiones que crearon estructuras socioeconómicas de desigualdad. Aunque América Latina es una de las regiones más desiguales del planeta, su población indígena es pobre entre los pobres y más vulnerable que cualquier otro grupo social. Según la CEPAL (2019), uno de cada cuatro latinoamericanos sufre pobreza crónica; una situación de carestía difícil de superar aún en entornos macroeconómicos favorables. Asimismo, los países con tasas más altas de pobreza crónica tienen también los índices más altos de pobreza indígena. Actualmente, en América Latina el número de hogares indígenas que viven en situación de pobreza duplica al número de hogares no indígenas; y, en el caso de la pobreza extrema, casi lo triplica (CEPAL, 2019). De lo anterior se desprende que superar la desigualdad y construir sociedades más prósperas y justas pasa por hacer de los pueblos indígenas sujetos de inclusión y justicia social.

El derecho a la educación también ha sido un reclamo importante en las luchas de los pueblos indígenas. En una primera etapa, la prioridad educativa se fijaba en la alfabetización, si bien manteniendo los gobiernos una estrategia de asimilación a la cultura y lengua hegemónicas. En la actualidad, los marcos jurídicos internacionales -especialmente el convenio 169 de la Organización Internacional del Trabajo (OIT), suscrito por la mayoría de los países latinoamericanos, y la Declaración de los Derechos de los Pueblos Indígenas de la Organización de las Naciones Unidas (ONU, 2007), han influido positivamente sobre las legislaciones nacionales y las políticas públicas relacionadas con los derechos de los pueblos indígenas. Algunos países han modificado las constituciones nacionales y emitido leyes de educación que reconocen la diversidad cultural y lingüística, y la identidad de los pueblos indígenas. En el caso de la educación, se han desarrollado alternativas como la educación intercultural bilingüe, e incluso algunos países como México, Ecuador y Bolivia han creado universidades basadas en este enfoque.

Pese a los avances antes mencionados, todavía existen diferencias entre la situación deseada y la realidad. Se observa, por ejemplo, que el acceso a la educación es menor y el analfabetismo más alto entre las poblaciones indígenas más rurales y aisladas, allí donde la presencia estatal es escasa o inexistente. Entre los grupos indígenas también existe una importante brecha de género, de tal manera que las tasas de analfabetismo y de deserción escolar son más elevadas entre las mujeres que entre los hombres. En relación con la pertinencia cultural, pese a los marcos normativos que protegen lengua y cultura, los datos señalan que la educación formal todavía es un agente amenazador en tanto que menos del 32\% de los indígenas siguen hablando una lengua indígena al finalizar la educación primaria, solamente el 5.3\% lo hace al terminar la secundaria, y apenas el 2\% al finalizar la Educación Superior (Banco Mundial, 2015). La situación antes mostrada es indicativa de lo complejo y difícil que resulta romper los esquemas pedagógicos tradicionales y los estigmas sociales de la discriminación asociados a las culturas indígenas.

$\mathrm{Al}$ analizar la situación educativa de la población 15 a 29 años se observa que, en la mayor parte de los países latinoamericanos, el porcentaje más alto de la población indígena de este grupo etario 
alcanza de 0 a 6 años de estudio, frente a los 7 años o más que alcanzan el resto de jóvenes (Del Popolo, López y Acuña, 2009). En el nivel superior, según datos aportados por SITEAL (2011) las probabilidades de que un graduado de nivel medio continúe estudios superiores son más bajas entre la población indígena de América Latina que el resto de habitantes. Se identifican en esta brecha tres situaciones específicas: a) Paraguay, donde la brecha étnica en el acceso a nivel superior y universitario trepa los 46 puntos; b) Perú, Ecuador y Chile, con brechas entre 16 y 19 puntos, y c) Bolivia, Brasil, Nicaragua y Panamá, donde la brecha es más reducida, pero continúa revelando desventajas, y Guatemala, donde no se registran diferencias en la proporción de graduados del nivel medio que acceden al nivel superior vinculadas a la condición étnica.

Considerando que la Educación Superior ha sido un espacio de difícil acceso para poblaciones vulnerables, la Conferencia Mundial de Educación Superior de la UNESCO recomendó el establecimiento y la garantía de condiciones no discriminatorias de acceso a la Educación Superior, que pudieran limitar el derecho de las personas a la Educación Superior por razones de religión, raza, sexo, idioma, económicas, sociales o culturales o incapacidad física (UNESCO, 2009). Como antes se mencionó, este marco normativo internacional y el empoderamiento ciudadano parecen haber favorecido el ingreso de la población indígena a la Educación Superior, de tal manera que la población estudiantil latinoamericana parece más matizada.

\section{2. ¿Quiénes son los estudiantes de educación de origen indígena?}

Diversos autores señalan que ha habido una cierta «irrupción» de los pueblos indígenas en la Educación Superior (Del Popolo, 2008; Zapata y Oliva, 2011), hecho que produce un cierto empoderamiento para el desarrollo de su propio recorrido académico. Al tiempo se continúan precisando medidas políticas sostenibles y adecuadas a las realidades de los estudiantes de todas las áreas, incluidos los que cursan estudios de educación (futuros maestros/as o profesionales de la educación) que es el ámbito que se trata en este estudio. Para ello, es necesario recopilar, comparar y analizar datos empíricos sobre características, necesidades y expectativas de estos estudiantes. Podemos encontrar algunos datos sobre el acceso y los servicios estudiantiles disponibles en fuentes administrativas, aunque estas fuentes a menudo están sesgadas o altamente fragmentadas. Así, en la actualidad, no es posible conocer concretamente el número de estudiantes universitarios de origen indígena en América Latina, ni sus condiciones de vida y sus posibilidades de éxito.

Asmar, Page y Radloff (2010) trazaron un perfil de estudiante indígena mayoritariamente femenino que accede a la universidad con más edad que sus compañeros no indígenas. Señalaron, asimismo, que el lugar de origen de estos estudiantes suele situarse a una distancia mayor del centro de estudios que el de sus compañeros no indígenas. Este elemento condiciona la ratio de estudiantes indígenas universitarios a tiempo completo (más baja que la de sus compañeros) y, además, en el caso latinoamericano, está frecuentemente conectado a regiones con mayor pobreza económica (López y Machaca, 2007).

De este modo, pese a que las condiciones de ingreso a la Educación Superior para los pueblos indígenas parecen haber mejorado (Rama, 2004), seguimos contando con estudios que reflejan cómo la comunidad indígena se encuentra infrarrepresentada (Paladino, 2009; López y Machaca, 2007), y en desventaja, planteándose como un reto conseguir que estos estudiantes consigan finalizar sus estudios universitarios (Pechenkina, Kowal y Paradies, 2011). Entre los factores clave que contribuyen al éxito 
de dichos estudiantes en sus estudios, Shah y Widin (2010) señalan la importancia de dar soporte a las escuelas y de realizar promoción de los estudios de Educación Superior.

Estudios previos en el contexto mexicano como el de Chávez-Arellano (2008) ya apuntó a que, si bien el origen étnico no condiciona el fracaso en los estudios, sí que genera otras adversidades relacionadas con la discriminación o con la soledad. Aspectos similares son también detectados en el contexto australiano por Asmar, Page y Radloff (2010), quienes también señalan que los estudiantes indígenas muestran un compromiso y una satisfacción con sus estudios iguales o superiores a las de sus compañeros no indígenas, aunque, sin embargo, tienen más posibilidades de considerar abandonar sus estudios. Estos elementos se añaden a otras dificultades que los propios estudiantes indígenas reportan como problemáticas y entre las que, por ejemplo, destacan el manejo del idioma en el que estudian (Chávez-Arellano, 2008), y el efecto que tienen los estereotipos sobre la convivencia universitaria (Ojeda y González, 2012).

Todas estas cuestiones han servido para plantear el debate acerca de cómo implicar en los estudios universitarios de manera más significativa a los estudiantes de origen indígena (Bradley, Noonan, Nugent y Scales, 2008) ya que, como Ossola (2010) evidenciaba en Argentina, pueden llegar a sentir la universidad como algo ajeno, un elemento que tiene consecuencias en los altos niveles de deserción y en las bajas tasas de graduación. De acuerdo a UNESCO-IESALC (2018), apenas en el 50\% de los estudiantes alcanza a obtener el título entre los 25 y 29 años.

Entre las posibles vías para conseguir mayor implicación, estudios anteriores se refieren a la puesta en marcha de programas que provean escenarios de sociabilidad (Czarny, 2010) y a trabajar para el reconocimiento de docentes indígenas (Paladino, 2009). Con ello, se podría contribuir a mejorar la incorporación al mercado laboral de la población indígena, un aspecto problemático en las sociedades latinoamericanas en la que, el porcentaje de indígenas que a igual calificación académica ocupan empleos de alto prestigio es menor que el porcentaje de no indígenas (CEPAL, 2019).

En nuestro estudio presentamos una caracterización del colectivo a través de los siguientes datos: a) la distribución por países de los estudiantes indígenas participantes; b) sus datos universitarios (nivel de estudios en curso, trayectoria académica y satisfacción con la experiencia universitaria); c) su contexto familiar; d) sus condiciones de vida, y, e) su participación académica y social. Por tanto, el presente trabajo pretende profundizar en el perfil sociodemográfico y en las condiciones de vida y de estudio, así como en la satisfacción con la vida universitaria de los y las estudiantes superiores del ámbito de la educación, de origen indígena, en el marco del proyecto TO-INN, con el propósito de aumentar la limitada información sobre los estudiantes de Educación Superior de pueblos indígenas y de contribuir a la reflexión sobre la dimensión social de la Educación Superior y los colectivos especialmente vulnerables.

\section{Método}

\subsection{Participantes}

Un total de 12238 estudiantes de 30 universidades de América Latina participaron de forma voluntaria en este estudio. Del total de la muestra, 7.974 (65,2\% del total) fueron mujeres, con una media de edad de 24,72años (D.T. = 7,58), y 4264 (34,8\%) eran hombres, con una edad media de 25,13 años (D.T. $=$ $7,62)$. Por otra parte, la submuestra de estudiantes pertenecientes a comunidades indígenas o pueblos 
originarios estuvo compuesta por un total de 702 personas, con una media de edad de 28,54 años (D.T. =9). De entre ellos, $296(42,2 \%)$ eran hombres, con una media de edad de 30,61 años (D.T. = 9,25), y $405(57,8 \%)$ eran mujeres, con una edad promedio de 27,02 (D.T. $=8,51)$.

Los criterios de inclusión para poder cumplimentar el cuestionario del estudio fueron: 1) ser mayor de edad, y 2) estar cursando algunos estudios superiores relacionados con el ámbito de la educación en el momento de contestar. Las características sociodemográficas de la muestra se desarrollan en el apartado de resultados de este trabajo.

\subsection{Instrumento}

Se diseñó un cuestionario online para analizarla Dimensión Social de la Educación Superior, concretamente en las instituciones que forman docentes y profesionales de la educación y se implementó entre las instituciones socias ya vinculadas al proyecto TO- INN a través de la plataforma Survey Monkey. El instrumento estuvo compuesto por un total de 34 ítems, distribuidos en las siguientes 4 dimensiones: a) Datos sociodemográficos (8 ítems categóricos); b) Datos universitarios (6 ítems categóricos); c) Vida y participación académica y social (3 ítems escalares y 14 categóricos), y d) Ámbito motivacional y expectativas de futuro (1 ítem escalar y1 categórico). Además, al final del cuestionario existía un 1 ítem abierto, en el que los participantes podían añadir cualquier información cualitativa respecto a su propia experiencia como estudiante de Educación. En un primer momento se realizó un estudio de validez de este instrumento, mediante su aplicación piloto con una submuestra compuesta por 299 estudiantes de 9 de las instituciones participantes en el proyecto. De este estudio piloto se desprendieron una serie de aspectos de mejora en relación con la composición de los ítems (se agruparon o desagregaron opciones de respuesta y se rediseñaron ítems que habían resultado problemáticos en la versión preliminar), el léxico y su aplicabilidad a cualquier contexto iberoamericano.

\subsection{Análisis de datos}

A partir del software Statistical Package for Social Sciences (SPSS, versión 22) para Windows se han realizado los análisis estadísticos que ha consistido en la obtención de indicadores descriptivos como el análisis de frecuencias (en el caso de los datos categóricos), y en la obtención de medias en caso de los ítems escalares, mediante el procedimiento Frecuencias, Medias y Tablas de contingencia. Para la comparación de variables escalares entre los estudiantes indígenas y el resto de la muestra, se empleó el análisis unifactorial de la varianza (oneway ANOVA).

\section{Resultados}

\subsection{Distribución muestral por país y pertenencia a comunidad indígena}

La tasa de respuesta por universidad fue irregular, dependiendo en muchos casos del tamaño de la institución. Por este motivo, se observó una gran variabilidad en el número de respuestas obtenidas en cada centro. El Gráfico1 resume la distribución por países de los estudiantes indígenas que participaron en el estudio, comparando su número con el de los estudiantes de esos mismos países que participaron en el estudio, pero no pertenecían a comunidades indígenas. En este punto se presenta la información de aquellos países que proporcionaron estudiantes originarios al estudio. 


\section{Gráfico 1. Estudiantes indígenas y no indígenas según país participante (en valor absoluto)}

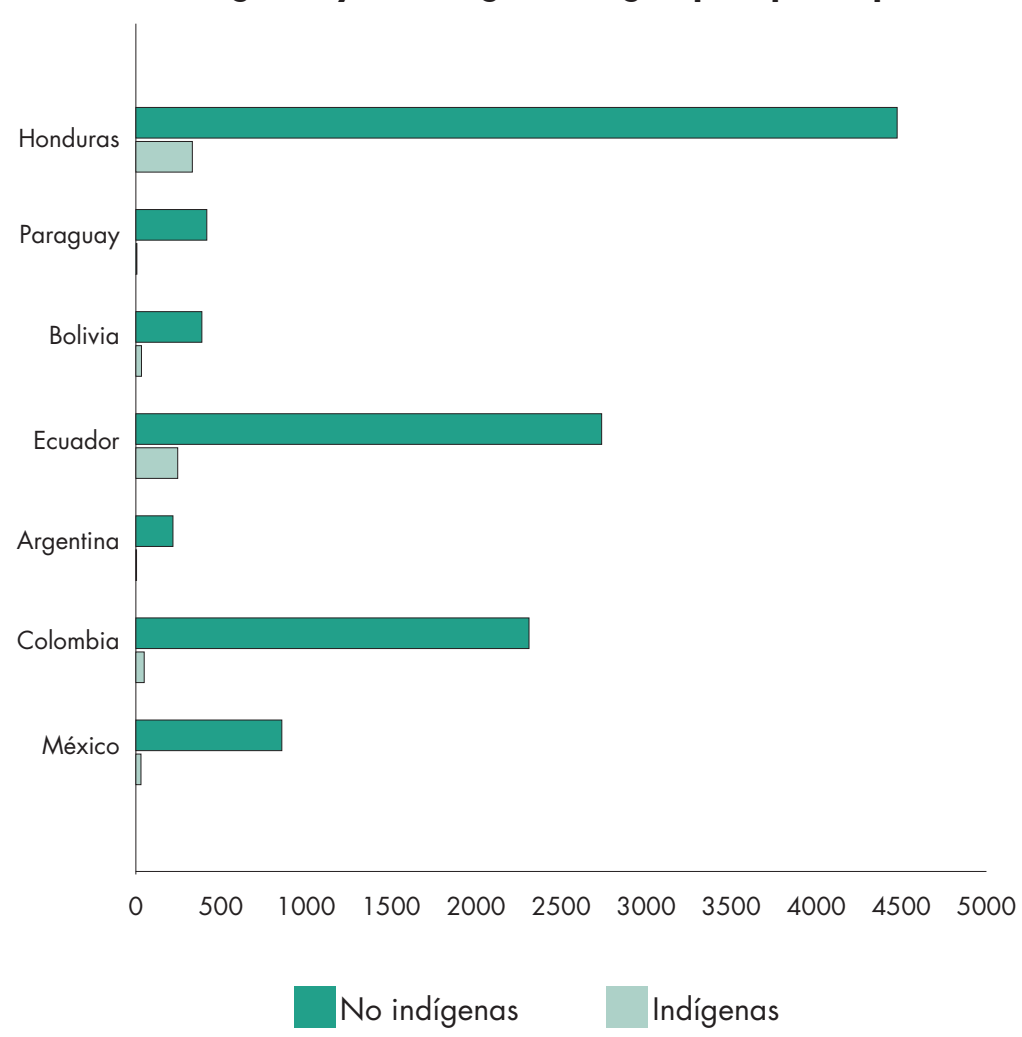

Fuente: elaboración propia.

\subsection{Datos universitarios}

Dentro del ámbito educativo, los estudios cursados más frecuentemente en nuestra muestra fueron los de Maestro/a de primaria o básica y los de Pedagogía o Ciencias de la Educación. La Tabla I resume de forma desagregada las titulaciones que estaban cursando los estudiantes indígenas que participaron en el estudio.

Tabla I. Estudios en curso (muestra completa)

\begin{tabular}{|c|c|c|}
\hline ESTUDIOS & $\mathrm{N}$ & PORCENTAJE \\
\hline Maestro/a de infantil, nivel inicial, parvulario o preescolar (0-6 años) & 20 & 2,9 \\
\hline Maestro/a de primaria o básica (6-12 años) & 206 & 29,4 \\
\hline Maestro/a de secundaria o enseñanza media (12-16 años) & 36 & 5,1 \\
\hline Profesor/a en Ciencias de la Educación (secundaria y terciaria) & 54 & 7,7 \\
\hline Psicopedagogía/Psicología de la Educación & 21 & 3 \\
\hline Educación, Pedagogía o Ciencias de la Educación & 184 & 26,2 \\
\hline Educación Social/Trabajo Social & 1 & 0,1 \\
\hline Educación Especial & 5 & 0,7 \\
\hline Enseñanza de Lengua extranjera & 50 & 7,1 \\
\hline Enseñanza de Educación Física & 3 & 0,4 \\
\hline Enseñanza Bilingüe & 43 & 6,1 \\
\hline Enseñanza de Artes & 3 & 0,4 \\
\hline Administración Educativa & 0 & 0 \\
\hline Posgrado y cursos/capacitaciones de especializaciones en educación & 21 & 3 \\
\hline Máster, Maestría o Magister en el campo de la Educación & 5 & 0,7 \\
\hline
\end{tabular}




\begin{tabular}{lccc}
\hline & ESTUDIOS & N & PORCENTAJE \\
\hline Máster de Formación del profesorado de secundaria & 2 & 0,3 \\
\hline Doccorado relacionado con la Educación & 0 & 0 \\
\hline Otros & 47 & 6,7 \\
\hline
\end{tabular}

Fuente: elaboración propia.

Cabe destacar que únicamente un $4 \%$ de estudiantes indígenas estaban realizando estudios de posgrado, así como el elevado número de personas que seleccionaron la opción «otros» (6,7\%), lo que revela la heterogeneidad de la nomenclatura y tipologías de estudios superiores en el ámbito de la Educación, causada por el gran número de países que participaron en el proyecto, y sus respectivas normativas.

Por otra parte, respecto al acceso a la universidad, la Tabla II resume las vías de acceso mediante las cuales los y las estudiantes indígenas accedieron a sus estudios superiores. Mayoritariamente lo hicieron vía selectividad o prueba propia de la universidad.

Tabla II. Vía de acceso a los estudios universitarios

\begin{tabular}{lccc}
\hline & VÍA DE ACCESO & N & PORCENTAJE \\
\hline Prueba de acceso general (selectividad o examen nacional) & 208 & 29,7 \\
\hline Prueba propia de acceso (por la universidad a la que se accede) & 236 & 33,7 \\
\hline Curso de nivelación & 14 & 2 \\
\hline Prueba de acceso para mayores de 25 años & 7 & 1 \\
\hline Prueba de acceso para mayores de 40 o 45 años & 10 & 1,4 \\
\hline Calificación de Formación Profesional & 69 & 9,8 \\
\hline Curso de Preparación Universitaria & 27 & 3,9 \\
\hline Acreditación de experiencia laboral & 61 & 8,7 \\
\hline Cambio de carrera & 26 & 3,7 \\
\hline Presentando el currículo vitae & 8 & 1,1 \\
\hline Solicitud de incorporación & 3 & 1,3 \\
\hline No hubo vía de selección & 3,7 \\
\hline
\end{tabular}

Fuente: elaboración propia.

Se incluyó una pregunta en relación con la tipología de centro de estudios de procedencia (público versus privado), para analizar posteriormente si esta variable se relaciona con la elección de titulaciones del ámbito de la educación y así determinar la homogeneidad de este colectivo de estudiantes. Mientras que el 77,4\% señaló que siempre había estudiado en instituciones públicas, solo un 6,8\% señaló haber cursado tanto la escolaridad básica como la secundaria en un centro privado. Por otro lado, un 15,8\% había estudiado o una u otra etapa educativa en una institución privada.

Otra de las cuestiones que se ha analizado son las condiciones de los estudiantes en los centros de Educación Superior, por lo general, estos estudiantes afirmaron tener una elevada satisfacción en relación con diversos aspectos de su vida universitaria (sobre 5): el contenido de las materias $(4,65)$, los compañeros y compañeras de clase $(4,37)$, la relación con el profesorado $(4,41)$, el trato recibido por la administración universitaria $(4,1)$, y la orientación recibida por parte de la universidad $(4,21)$. El nivel de satisfacción general con la vida de estudiante fue de 4,4 en esta misma escala. 


\subsection{Contexto familiar}

Respecto al nivel socio-educativo de los padres y las madres de los encuestados, el más frecuente fue el de estudios primarios (un 38,7\% en el caso de las madres y un 39,4\% en de los padres). Resulta relevante también el porcentaje de madres $(22,5 \%)$ y padres $(18,3 \%)$ que no disponían de estudios básicos. Únicamente un 7,3\% de las madres de las personas encuestadas tenían estudios universitarios, por un 9,9\% de los padres. El siguiente Gráfico resume la ocupación de los progenitores en el momento de contestar el cuestionario, desagregada por género.

Gráfico 2. Perfil socio-laboral de los progenitores (en valores absolutos)

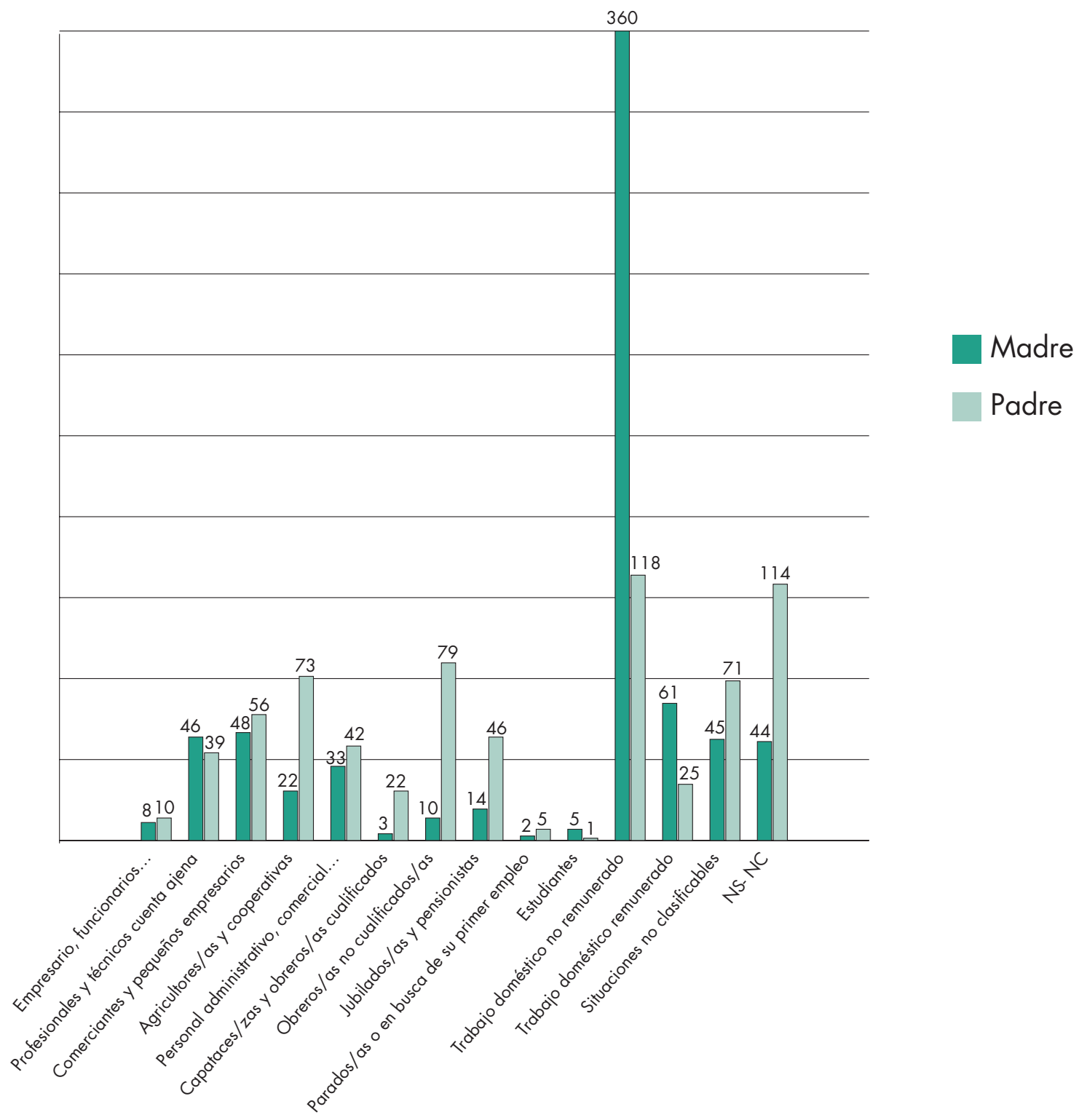

Fuente: elaboración propia. 
En relación con la ocupación de las madres, los participantes informaron de que un 51,4\% realizaba tareas del hogar no remuneradas, un 10,1\% tenían empleos de baja cualificación, un 11,1\% desempeñaba tareas técnicas vinculadas al sector comercio, un 6,6\% eran profesionales medios y un 1,1\% eran empresarias. En el caso de los padres, un 6,6\% eran jubilados o pensionistas, un 14,9\% realizaba tareas de baja cualificación, el 27,5\% llevaba a cabo tareas técnicas vinculadas al sector comercio o a la agricultura, un 5,6\% eran profesionales medios y un 1,4\% empresarios.

\subsection{Condiciones de vida}

Respecto a las condiciones de vida de estos estudiantes, resulta especialmente destacable que un $55,5 \%(\mathrm{n}=389)$ de los y las estudiantes indígenas afirmaron tener personas dependientes a su cargo, muy por encima del $34,5 \%$, que representaba el porcentaje de estudiantes en esta situación sobre la muestra global de América Latina. Por otra parte, un 45,8\% de participantes declararon haber tenido que desplazar su residencia de una zona rural a una urbana para poder realizar sus estudios. El Gráfico 3 resume la situación residencial de estas personas en el momento de contestar al cuestionario. La satisfacción media subjetiva con la situación residencial de estas personas fue de 3,46 (D.T. = 1,25), en una escala entre 1 (completamente insatisfecho/a) y 5 (máxima satisfacción).

\section{Gráfico 3. Situación residencial (porcentaje)}

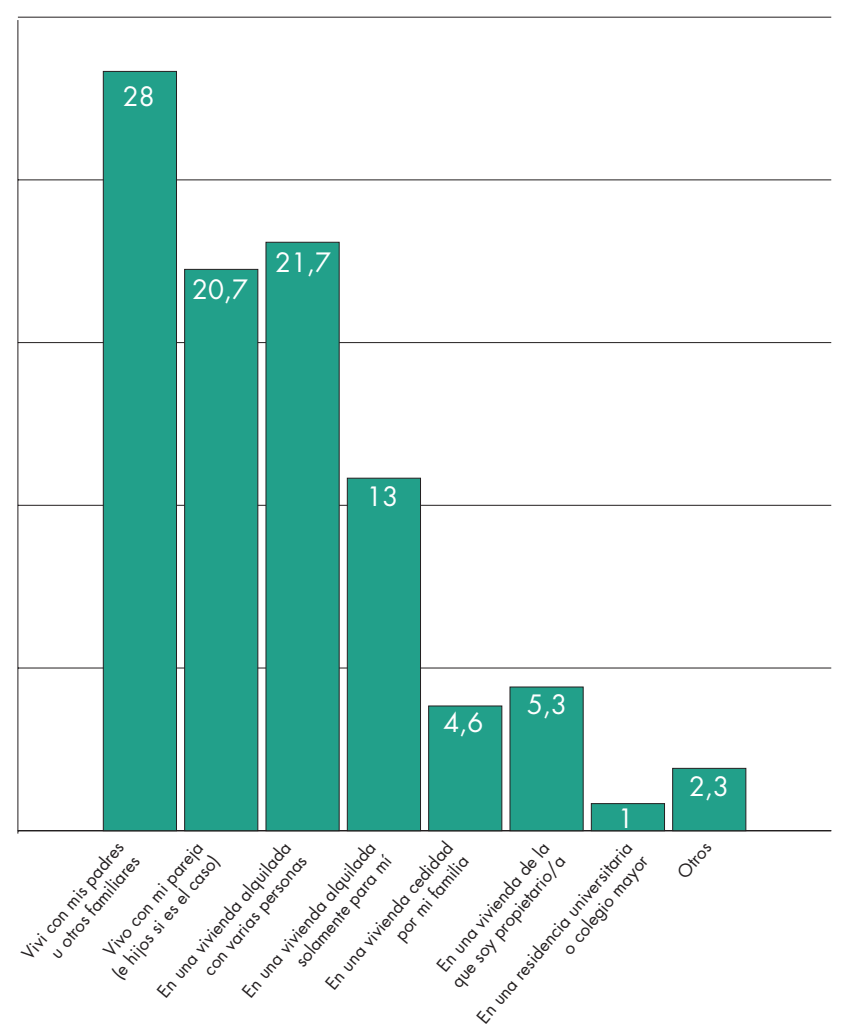

Fuente: elaboración propia.

Por otra parte, la diferencia en el tiempo medio dedicado en desplazamientos a la universidad a diario entre la muestra de estudiantes indígenas y el resto fue estadísticamente significativa, $(\mathrm{F}=131,08$ : $\mathrm{P}<005)$. Los primeros dedicaban 123 minutos (D.T. $=141,46)$ a los desplazamientos, mientras que los segundos 84,23 (D.T. $=84,53)$. 
En cuanto a la situación laboral de los estudiantes indígenas, un 38,1 \% afirmó no realizar ninguna actividad remunerada mientras estudiaba. De entre aquellos que sí trabajaban, un 43,7\% lo hacían en un empleo relacionado con el ámbito de la Educación: con una dedicación superior a 30 horas semanales $(21,4 \%)$, de entre 15 y 30 horas semanales (11,5\%), o de menos de 15 horas (10,8\%). El principal motivo al que aludieron los y las estudiantes indígenas para trabajar al mismo tiempo que estudiaban fue «prepararme para la futura profesión, o supone una formación complementaria», (puntuación media de 4,46 en una escala de acuerdo entre 1 y 5 puntos), seguido de «Tengo ganas de hacer algo práctico y tener otras experiencias profesionales» (puntuación media de 4,39). La necesidad de una fuente de ingresos para financiar los estudios fue la tercera opción que recibió una puntuación más alta (4,33). En cuanto a la forma en que los y las estudiantes indígenas participantes financiaban sus estudios, la más habitual consistía en los ingresos del trabajo durante el curso (42\%), ya fuera fijo u ocasional, seguido por la ayuda financiera de los padres (33,9\%). Únicamente un 7,7\% de los estudiantes recibían becas o ayudas al estudio.

\subsection{Participación académica y social}

Como se observa en la siguiente Tabla, la participación en la vida universitaria de las personas entrevistadas fue, por lo general, baja, a excepción del 21,1\% de personas que afirmaron acudir a grupos artísticos o deportivos. Del mismo modo, resulta destacable que únicamente un 16,5\% de estos/as estudiantes hubieran votado en elecciones de representantes estudiantiles.

Tabla III. Participación universitaria

\begin{tabular}{|c|c|}
\hline ÁMBITO DE PARTICIPACIÓN & PORCENTAJE \\
\hline Representante estudiantil órgano de Facultad & 4 \\
\hline Representante estudiantil Gobierno universidad & 1,9 \\
\hline Asamblea y/o sindicato de estudiantes & 5,4 \\
\hline Asociaciones estudiantiles & 7,4 \\
\hline Votar en las elecciones de representantes estudiantiles & 16,5 \\
\hline Manifestaciones $y / 0$ huelgas & 6 \\
\hline Voluntariado promovido por la universidad & 11,8 \\
\hline Grupos de investigación o innovación & 10,4 \\
\hline Grupos artísticos o de deporte & 21,1 \\
\hline Delegado/a de curso & 5,6 \\
\hline Otros & 2,6 \\
\hline
\end{tabular}

Fuente: elaboración propia.

El siguiente Gráfico resume la frecuencia de uso de los servicios e instalaciones universitarias por parte de los y las estudiantes indígenas que contestaron al cuestionario. 


\section{Gráfico 4. Frecuencia de uso de instalaciones y servicios universitarios (porcentaje)}

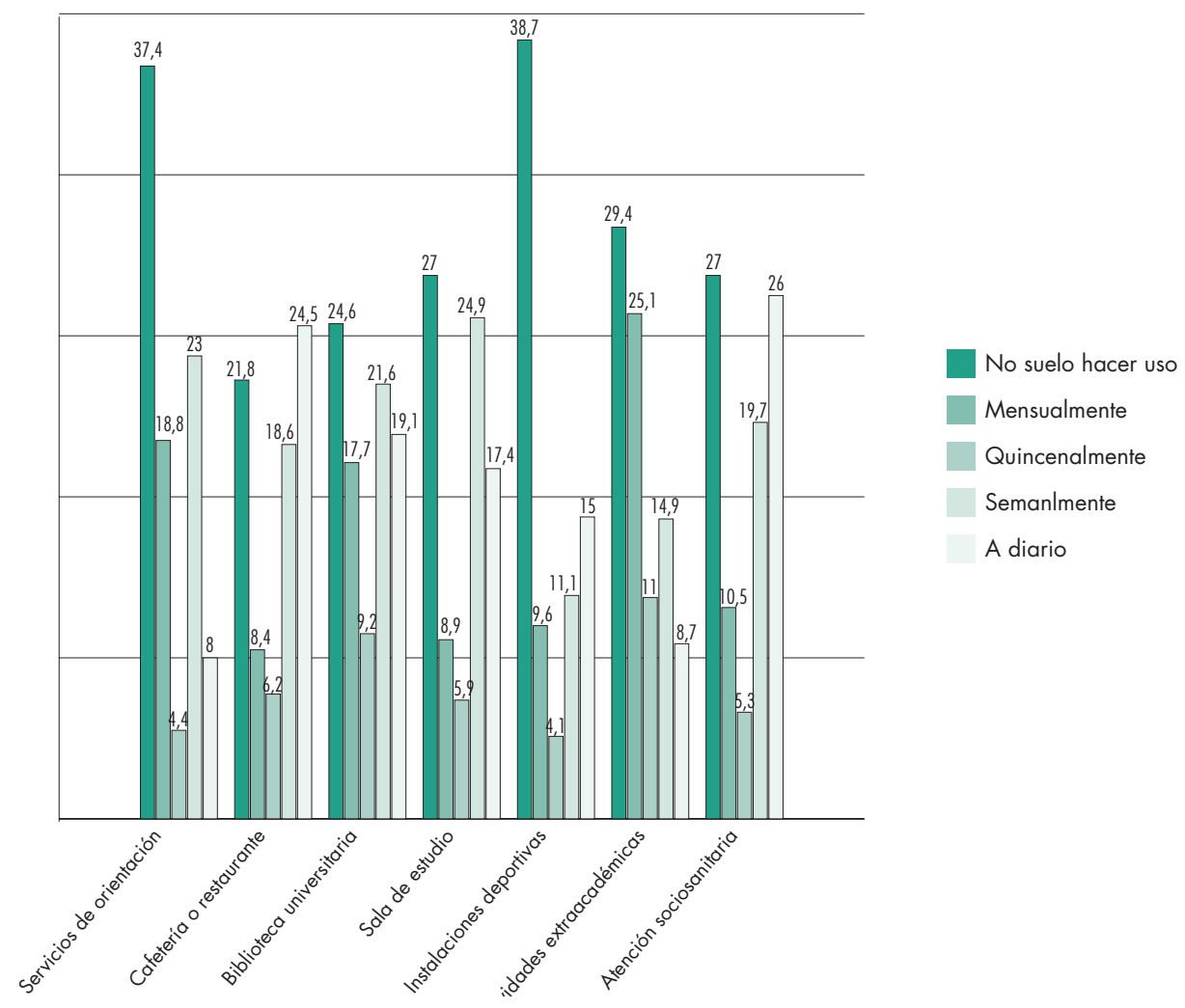

Fuente: elaboración propia.

No obstante, la participación de estos estudiantes en actividades fuera de la universidad y la frecuencia con que lo hacía, era superior a la que mostraban en la universidad. Un 22\% afirmó participar en actividades culturales, un $13,4 \%$ a organizaciones juveniles, un 16,5\% en clubes deportivos, un $15,5 \%$ en asociaciones de tipo religioso, un $10,8 \%$ en ONG o asociaciones de voluntariado, y un 9,7\% afirmó pertenecer a algún partido político. En el otro extremo, los ámbitos de participación menos frecuentes fueron las asociaciones vecinales $(4,6 \%)$, y los sindicatos o asociaciones profesionales $(2,4 \%)$.

\section{Discusión y conclusiones}

A pesar de las limitaciones de tipo metodológico de este trabajo, como la gran diferencia de tamaño entre las muestras analizadas, y de la gran diversidad de normativas existentes en los países participantes en el proyecto, nuestros resultados permiten caracterizar de manera genérica a los y las estudiantes de Educación que pertenecen a comunidades indígenas o pueblos originarios, así como acercarnos a sus condiciones de vida y trayectoria personal y académica. Nuestros datos reflejan una situación socio-educativa de los estudiantes indígenas menos ventajosa en comparación con la de sus compañeros no indígenas, y refrendan lo que estudios anteriores habían referido para este grupo de población en el contexto analizado, América Latina (Banco Mundial, 2015; Mato, 2015).

Respecto al género, se observa que, aunque hay más mujeres indígenas que hombres estudiando titulaciones relacionadas con el ámbito de la Educación (un ámbito disciplinar feminizado), el porcentaje de mujeres indígenas en dichos estudios es sustancialmente inferior al de mujeres no indígenas. Una explicación posible a esta diferencia podría tener que ver con el limitado acceso de los varones indígenas a estudios universitarios académicamente más demandantes y considerados de mayor prestigio social en Améri- 
ca Latina, como son la medicina ola ingeniería. Respecto a la edad, tanto en el caso de los hombres como de las mujeres, esta resultó ser significativamente superior en el colectivo de estudiantes de procedencia indígena que en el resto de la muestra que nos remite a un indicio de problemas relacionados con la reprobación, el abandono y la retención y que ya había reflejado en estudios anteriores (UNESCO-IESALC, 2018). Las carreras del ámbito educativo que estas personas cursan con mayor frecuencia fueron la de Maestro/a de educación básica (de 6 a 12 años) o Pedagogía, y un porcentaje bajo accede a formaciones de segundo ciclo. Este dato que hay que continuar analizándolo en profundidad nos puede estar avisando de la no continuidad del colectivo en formaciones de segundo o tercer ciclo, quizás la teoría credencialista pueda explicar algunas de estas consecuencias.

Se puede afirmar que, si bien, por lo general la satisfacción de los estudiantes con su vida universitaria y con los servicios recibidos por sus instituciones, la participación en el ámbito universitario era realmente baja a nivel de gobernanza y gestión universitaria, si bien declaran hacer un uso bastante frecuente de los servicios que les ofrece su centro universitario. Esta situación pone de manifiesto el predominio de los valores de la cultura hegemónica en los distintos países y las dificultades de los estudiantes indígenas para adaptarse a patrones de vida y convivencia distintos a los de su cultura de origen (Chávez-Arellano, 2008). Por otro lado, este dato resulta contradictorio con el hecho de que los participantes en nuestro estudio, fuera de la universidad, participan en mucha mayor medida en diferentes ámbitos sociales como asociaciones artísticas, clubes deportivos, o partidos políticos, entre otros.

En cuanto a su situación residencial, la mayor parte de estos y estas estudiantes viven con sus familiares o bien compartiendo vivienda, estando por lo general bastante satisfechos con su situación residencial. No obstante, conviene recordar que muchos estudiantes indígenas son migrantes, proceden de comunidades rurales y comparten viviendas precarias, en muchos casos en zonas pobres de los asentamientos urbanos. En este sentido, se observó una gran diferencia a nivel del tiempo que los estudiantes indígenas debían emplear a diario para llegar a su centro de estudios (más de dos horas en promedio), siendo muy superior al que dedicaban los y las estudiantes no pertenecientes a pueblos indígenas (Asmar, Page y Radloff, 2010). Este elemento indica una mayor dispersión geográfica de los primeros e incrementa la dificultad para seguir sus estudios. En cuanto al trabajo, más de la mitad de los estudiantes indígenas encuestados trabajaba en el sector educativo (con diferentes niveles de dedicación horaria), con la principal motivación de prepararse para el trabajo futuro, pero también para poder costearse los estudios. Continuando con la comparación entre ambas submuestras, resulta especialmente destacable el hecho de que el número de estudiantes indígenas que se ocupaban de personas dependientes (un 55,4\%) era muy superior al de estudiantes no indígenas en esta situación (un 35,7\%). Esta diferencia podría tener relación con los niveles socioeducativos y de pobreza que las familias y progenitores de los estudiantes indígenas vive.

De cara al futuro, resultaría interesante profundizar en las diferencias entre el perfil de estudiantes indígenas de Educación, especialmente mediante la obtención de una muestra más amplia que facilite la comparabilidad y representatividad de los datos. También resultaría pertinente, ahondar en los motivos por los que estas personas de origen indígena escogieron este tipo de estudios, para intentar establecer perfiles motivacionales de los estudiantes de Educación, el papel de la lengua y la cultura en las trayectorias estudiantiles, así como para examinar la hipotética relación entre estos perfiles motivacionales, su trayectoria personal y académica, y las actividades de participación académica y social en que participan, ya sea mediante estudios cuantitativos, desde una aproximación cualitativa o desde una perspectiva mixta. Por otra parte, debido a la elevada cantidad de datos de que dispone el proyecto, también resultaría 
interesante profundizaren otras realidades, tal vez poco exploradas, como las condiciones de vida de los estudiantes con diversidad funcional.

En 2015 había casi 23 millones de estudiantes matriculados en las instituciones de educación terciaria (UNESCO-IESALC, 2018) y este aumento en el acceso formal a la universidad puede ser entendido como un avance muy importante, pero es preciso diferenciar la distancia entre acceder a la universidad y garantizar el derecho a la Educación Superior, y asegurar condiciones adecuadas que permitan una inclusión real, una participación auténtica y el logro de aprendizajes pertinentes y de calidad.

Las universidades iberoamericanas, en las últimas décadas, han experimentado profun-dos cambios quelas han obligado, en muchos casos, a reestructurar sus instituciones y replantear sus funciones y servicios. Además de replantear sus procesos de innovación institucional. En este contexto, la dimensión social evidencia el imperativo de conside-rar el colectivo de estudiantes, sus características, condiciones de vida y participación y sus expectativas. En este sentido, es preciso analizar la pertinencia de las universidades como instituciones inclusivas, comprometidas con todos los/las estudiantes y, especial-mente, con los colectivos más vulnerables.

En este proceso detectamos una ausencia de datos fehacientes, estudios recientes o datos actualizados sobre aspectos sociodemográficos, datos universitarios, condiciones de vida y de participación académica y social y motivaciones o expectativas de futuro de los/las estudiantes. Es por ello que consideramos que el cuestionario sobre la dimensión creado en el contexto TO-INN puede ser de gran utilidad para la comunidad académica y educativa.

\section{Referencias bibliográficas}

Ariño, Antonio (2014): “La dimensión social de la Educación Superior”. Revista de Sociología de la Educación RASE, 7 (1), 17-41.

Asmar, Christine; Page, Susan y Radloff, Ali (2011): “Dispelling myths: Indigenous students' engagement with university”. AUSSE Research Briefings, 10, 1-16.

Banco Mundial (2015). Latinoamérica Indígena en el siglo XXI. Washington, D.C.: Banco Mundial.

Bradley, Denise; Noonan, Peter; Nugent, Helen y Scales, Bill (2008). Review of Australian higher education: Final Report. Canberra, ACT: Department of Education, Employment, and Workplace Relations, Australian Government.

CEPAL (2014). Los pueblos indígenas en América Latina. Avances en el último decenio y retos pendientes para garantía de sus derechos (en línea). https://repositorio.cepal.org/bitstream/handle/11362/37050/4/S1420783_es.pdf.

CEPAL (2019). Panorama Social de América Latina. Santiago: CEPAL.

Convenio Núm. 169 de la OIT sobre pueblos indígenas y tribales en países independientes (2014). Declaración de las Naciones Unidas sobre los Derechos de los Pueblos Indígenas (en línea). https://www. mop.cl/asuntosindigenas/Documents/convenio169.pdf.

Chávez-Arellano, María Eugenia. (2008): "Ser indígena en la Educación Superior ¿desventajas reales o asignadas?”. Revista de la Educación Superior, 37 (148), 31-55. 
Czarny, Gabriela (2010): “Jóvenes indígenas en la Universidad Pedagógica Nacional. México: Relatos de experiencias en Educación Superior”. Inclusión Social y Equidad en la Educación Superior ISEES, 7, 39-59.

Del Popolo, Fabiana; López, Mariana y Acuña, Mario. (2009). Juventud indígena y afrodescendiente en América Latina: inequidades sociodemográficas y desafios de políticas. España: CELADE-CEPAL.

Del Popolo, Fabiana (2008). Los pueblos indígenas y afrodescendientes en las fuentes de datos: experiencias en América Latina. Santiago de Chile: CEPAL.

Didou, Silvye (2006): "Acción afirmativa y Educación Superior en América Latina: debate en ciernes y estrategias emergentes" en Pilar Díaz-Romero (Ed.). Caminos para la Inclusión en la Educación Superior. Perú: OEI- Fundación Equitas.

Henríquez, Pedro (2018). Tendencias de la Educación Superior en América Latina y el Caribe 2018. Córdoba: Instituto Internacional de la UNESCO para la Educación Superior en América Latina y el Caribe. UNESCO, IESALC y UNC.

IDEA (2007). ¿Los de afuera? La exclusión social en América Latina. Banco Interamericano de Desarrollo Departamento de Investigación (en línea). https://www.iadb.org/es/noticias/articulos/2007-11-02/ los-de-afuera-la-exclusion-social-en-america-latina,4119.html.

López, Luis Enrique y Machaca, Guido (2007): “Acceso indígena a la Educación Superior en Chile y Perú: Un desafío para las políticas de equidad". Inclusión Social y Equidad en la Educación Superior ISEES, 2, $13-62$.

López, Luis Enrique y Küper, Wolfgang (1999): "La educación intercultural bilingüe en América Latina: balance y perspectivas". Revista Iberoamericana de Educación, 20, 17-85.

Mato, Daniel (2015): "Educación Superior y pueblos Indígenas en América Latina: contextos y experiencias” en Daniel Mato (Coord.). Educación Superior, Estados y pueblos indigenas en América Latina. Contextos, experiencias, conflictos y desafíos. Buenos Aires: Universidad Nacional Tres de Febrero.

Mato, Daniel. (Coord.) (2008). Diversidad Cultural e Interculturalidad en Educación Superior. Experiencias en América Latina. Caracas: IESALC-UNESCO.

ONU (2007). Declaración de las Naciones Unidas sobre los derechos de los pueblos indígenas (en línea). https://www.un.org/development/desa/indigenous-peoples-es/declaracion-sobre-los-derechos-delos-pueblos-indigenas.html.

Ossola, María Macarena (2010): "Pueblos indígenas y Educación Superior. Reflexiones a partir de una experiencia de jóvenes wichí en la Universidad Nacional de Salta”. Inclusión Social y Equidad en la Educación Superior ISEES, 8, 87-105.

Paladino, Mariana. (2009): "Pueblos indígenas y Educación Superior en la Argentina. Datos para el debate”. Inclusión Socialy Equidad en la Educación Superior ISEES, 6, 81-122.

Pechenkina, Ekaterina; Kowal, Emma y Paradies, Yin. (2011): “Indigenous Australian Students' Participation Rates in Higher Education: Exploring the Role of Universities". The Australian Journal of Indigenous Education, 40, 59-68. https://doi.org/10.1375/ajie.40.59. 
Rama, Claudio (2004): "La cuestión de la Educación Superior indígena en América Latina" en Avelina Pancho (Coord.). Educación Superior indígena en Colombia: una apuesta de futuro y esperanza. Bogotá: UNESCO/IESALC.

Robles, Claudia (2009): "Pueblos indígenas y programas de transferencias con corresponsabilidad. Avances y desafíos desde un enfoque étnico". Serie Politicas Sociales, 156, 3-57.

Shah, Mahsood y Widin, Jacquie (2010): “Indigenous Students' Voices: Monitoring Indigenous Student Satisfaction and Retention in a Large Australian University". Journal of Institutional Research, 15 (1), 28-41.

SITEAL (2011). Informe sobre tendencias sociales y educativas en América Latina 2011: La educación de los pueblos indigenas y afrodescendientes. Buenos Aires: IIPE-UNESCO.

UNESCO - IESALC (2018). Tendencias de la Educación Superior en América Latina y el Caribe. Caracas: Instituto Internacional de la UNESCO para la Educación Superior en América Latina.

UNESCO (2009). Conferencia Mundial sobre la Educación Superior 2009: La nueva dinámica de la Educación Superior y la investigación para el cambio social y el desarrollo (en línea). http://www.unesco. org/education/WCHE2009/comunicado_es.pdf.

Zapata, Claudia y Oliva, María Elena (2011): "Experiencia de inserción e impacto institucional de los becarios del Programa Internacional de Becas de la Fundación Ford con ascendencia y adscripción indígena en la Universidad de Chile". Inclusión Social y Equidad en la Educación Superior ISEES, 9, 43-71.

\section{Notas biográficas}

Jorge Luis Méndez-Ulrich es Licenciado en Psicología por la Universitat Autònoma de Barcelona y Doctor en Psicología por esta misma Universidad. Actualmente es profesor del Departamento de Métodos de Investigación y Diagnóstico en Educación de la Facultad de Educación de la Universitat de Barcelona y del Departamento de Psicología Básica, Evolutiva y de la Educación de la Facultad de Psicología de la Universitat Autònoma de Barcelona, así como consultor del Grado en Psicología de la Universitat Oberta de Catalunya. De 2014 a 2015 ha sido miembro del Comisionado del Rector para Participación, Empleabilidad y Emprendimiento social de la UB, donde ha desarrollado diversos proyectos relacionados con la participación de toda la comunidad universitaria, así como de empleabilidad de estudiantes, PDI y PAS.

Alejandra Montané López es Doctora en Pedagogía, licenciada en Filosofía y Ciencias de la Educación. Profesora del Departamento de Didáctica y Organización Educativa de la Universidad de Barcelona. Coordinadora del proyecto TO INN, From tradition to Innovation in Teacher Training Institutions financiado por el programa Esasmus+ Acción K2 Capacity Building referencia (573685-EPP-2016-1ES-EPP KA2-CBHE-JP). Es miembro del grupo de investigación e innovación educativa FODIP de la Universidad de Barcelona. Ha participado en proyectos internacionales entre los que cabe destacar la Red RIAPE (Red Iberoamericana de Investigación de Políticas Educativas). Es autora de numerosos artículos relacionados con políticas educativas y formación del profesorado.

Juan Llanes Ordóñez es Profesor lector en el Departamento de Métodos de Investigación y Diagnóstico en Educación de la Facultad de Educación de la Universitat de Barcelona. Doctor en Educación y 
